\title{
Angiotensin converting enzyme inhibitors remain the first treatment of choice
}

\author{
Pyung Chun $\mathrm{Oh}^{1,2}$, Ichiro Sakuma ${ }^{3}$, Toshio Hayashi ${ }^{4}$, and Kwang Kon $\mathrm{Koh}^{1,2}$
}

\author{
${ }^{1}$ Division of Cardiology, \\ Department of Internal Medicine, \\ Heart Center, Gachon University \\ Gil Medical Center, Incheon; \\ ${ }^{2}$ Gachon Cardiovascular Research \\ Institute, Incheon, Korea; \\ ${ }^{3}$ Cardiovascular Medicine, \\ Hokko Memorial Clinic, Sapporo; \\ ${ }^{4}$ Department of Geriatrics, \\ Nagoya University Graduate \\ School of Medicine, Nagoya, \\ Japan
}

\section{See Article on Page 267-276}

Angiotensin converting enzyme (ACE) inhibitors (ACEIs) are beneficial and are strongly recommended for patients with acute myocardial infarction (MI) associated with heart failure, left ventricular systolic dysfunction, or preserved left ventricular systolic function. A number of clinical trials have demonstrated the benefits of ACEIs in such patients $[1,2]$. In contrast, several clinical trials have consistently failed to demonstrate the benefits of angiotensin receptor blockers (ARBs). In addition, the proposed ARB-MI paradox led to a certain amount of caution in the use of ARBs because several meta-analyses suggested that ARBs do not prevent MI or cardiovascular death, in contrast to ACEIs $[3,4]$. However, this hypothesis is now considered to be misleading.

Using propensity score matching, Lee et al. [5] investigated the association between treatment with ARBs and clinical outcomes in patients without left ventricular dysfunction or heart failure after acute MI who had a ventricular ejection fraction $\geq 40 \%$. The effect of ARBs on in-hospital mortality and 12-month major adverse cardiovascular events (MACEs) was assessed using matched logistic and Cox regression models. Compared with ACEIs, ARBs significantly reduced in-hospital mor- tality (1.3\% vs. 3.3\%; hazard ratio [HR], 0.379; 95\% confidence interval [CI], 0.190 to $0.756 ; p=0.006$ ) and 12 -month MACEs (4.6\% vs. 6.9\%; HR, o.661; $95 \%$ CI, 0.457 to $0.956 ; p=0.028)$. However, the benefit of ARBs on 12-month mortality compared with ACEIs was marginal (4.3\% vs. $6.2 \%$; HR, 0.684; $95 \% \mathrm{CI}$, 0.467 to $1.002 ; p=0.051)$. The authors concluded that ARBs are not inferior to, and may be better than, ACEIs in Korean patients with acute MI.

The study is of note because it differs from the current American and European guidelines. Although Asians, including Koreans and Japanese, suffer more frequently from uncomfortable dry coughs when undergoing treatment with ACEIs in comparison to Caucasians, the significantly reduced in-hospital mortality and 12-month MACEs with ARBs must also be considered. We have several questions to ask of this study. What about other similar studies from Korea? What is the mechanism of ARBs compared to ACEIs that would lead to these different outcomes? Indeed, do the mechanisms actually differ? Or, were the results of this study caused by an unexplained bias, which has been detected in many other clinical studies, including a number of well-performed studies?

Yang et al. [6] reported a study based on 53 Korean hospitals involved in the 
treatment of acute MI. They investigated the association between ARBs treatment and clinical outcomes in patients with ST segment elevation MI who underwent primary percutaneous coronary intervention and had a left ventricular ejection fraction $\geq 40 \%$ [6]. This study was very similar to that by Lee et al. [5] with regard to the study period and characteristics of the participants, and also because it was a prospective cohort study which used data from a nationwide large-scale registry. However, this study did not include a group of patients who had not received a rennin-angiotensin system (RAS) blocker. The study demonstrated that cardiac death or MI occurred in 21 patients (1.8\%) in the ARBs group and 77 patients (1.7\%) in the ACEIs group. After propensity score matching, no significant difference was identified in the rate of cardiac death or MI between the ARBs and ACEIs groups (21 [1.8\%] vs. 23 [2.0\%]; adjusted HR, 0.65; 95\% CI, 0.30 to 1.38; $p=0.65)$.

The RAS is involved in many of the stages in developing atherosclerosis. Endothelial dysfunction is one of the first steps in the development of overt atherosclerosis. ACE inactivates kinins produced within the endothelium. Thus, ACE inhibition could increase endothelial bradykinin levels, resulting in the stimulation of nitric oxide (NO) synthase via the activation of $\mathrm{B} 2$ receptors on the endothelial cell surface and also in the activation of phospholipase A2 and the release of vasodilating prostaglandins from the cell membrane (Fig. 1). In addition, ACEIs may induce cough in sensitive patients, such as Asians, by increasing the bradykinin levels. Alternative-

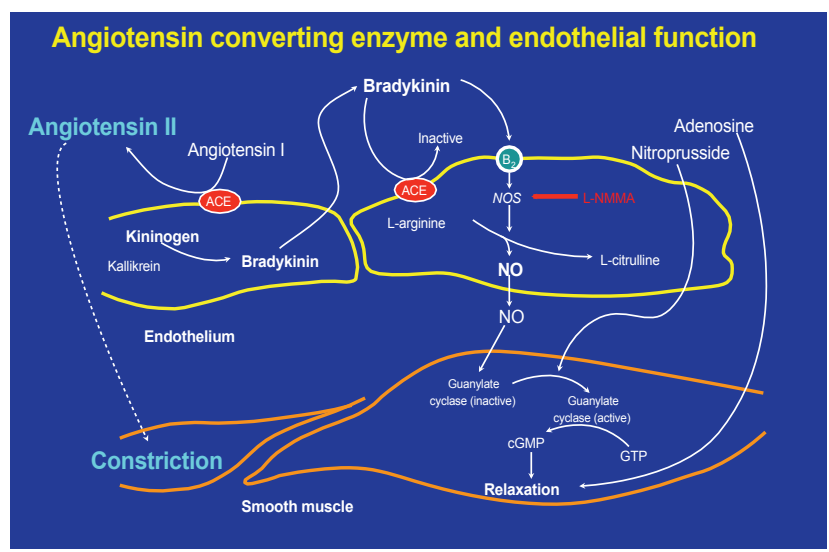

Figure 1. Angiotensin converting enzyme (ACE) and endothelial function. NOS, nitric oxide synthase; L-NMMA, NG-methyl-L-arginine; NO, nitric oxide; cGMP, cyclic guanosine monophosphate; GTP, guanosine triphosphate.

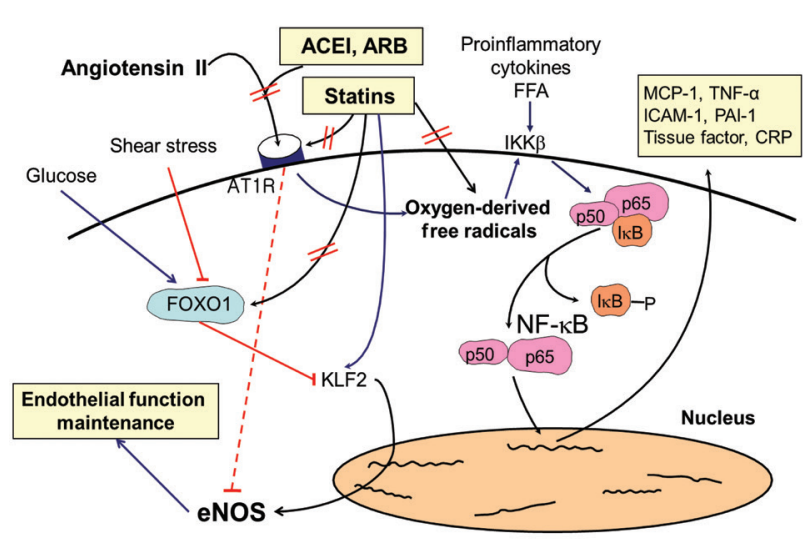

Figure 2. Additive/synergistic beneficial effects of angiotensin converting enzyme (ACE) inhibitors or angiotensin receptor blockers (ARBs) and statins on both endothelial function and insulin sensitivity. Dysregulation of the rennin-angiotensin system (RAS) contributes to the pathogenesis of atherosclerosis. Angiotensin II binds to angiotensin II type I receptor $\left(A T_{1} R\right)$ resulting in the enzymatic production of oxygen-derived free radicals. Free fatty acids (FFA) also promote oxygen-derived free radical generation in vascular endothelial cells and smooth muscle cells. This leads to dissociation of inhibitory factor IkB, with the subsequent activation of nuclear transcription factor $(\mathrm{NF}-\kappa \mathrm{B})$, which stimulates the expression of proinflammatory genes, chemokines, and cytokines. Importantly, elevated levels of FFA associated with insulin resistance, obesity, diabetes mellitus, and metabolic syndrome cause endothelial dysfunction by activating innate immune inflammatory pathways upstream of NF- $\mathrm{B}$. Thus, inflammation and oxidative stress contribute to endothelial dysfunction and insulin resistance while endothelial dysfunction and insulin resistance promote oxidative stress and inflammation. This demonstrates the reciprocal relationship between insulin resistance and endothelial dysfunction. Statins down-regulate the expression of $\mathrm{AT}_{1} \mathrm{R}$ via reduction of low-density lipoprotein cholesterol levels. Krüppel-like factor 2 (KLF2) is implicated as a key molecule for maintaining endothelial function. High-glucose-induced, forkhead box $\mathrm{O}_{1}$ (FOXO1)-mediated KLF2 suppression is reversed by statins. Furthermore, experimental studies have shown a cross-talk between hypercholesterolemia and RAS at multiple stages. Accordingly, combined therapy with statins and RAS inhibitors show additive/synergistic beneficial effects on endothelial dysfunction and insulin resistance when compared with monotherapy in patients with cardiovascular risk factors by both distinct and interrelated mechanisms. Reproduced with permission from Lim et al. [15] and Koh et al. [16]. ACEI, ACE inhibitor; IKK $\beta$, I kappa B kinase- $\beta$; MCP, monocyte chemoattractant protein; TNF- $\alpha$, tumor necrosis factor- $\alpha$; ICAM-1, intercellular adhesion molecule-1; PAI-1, plasminogen activator inhibitor-1; CRP, C-reactive protein; eNOS, endothelial nitric oxide synthase. 
ly, ACE inhibition could reduce the intracellular production of superoxide anions via the reduced activity of angiotensin II-dependent nicotinamide adenine dinucleotide/nicotinamide adenine dinucleotide phosphate oxidase systems within the endothelium $[7,8]$. Thus, ACEIs therapy may retard the progression and acute manifestations of atherosclerosis by increasing intravascular NO bioactivity and preventing the activation of nuclear transcription factor (NF- $\mathrm{kB}$ ) with its potential proinflammatory consequences. In this regard, we demonstrated that quinapril therapy improves NO bioactivity with enhanced endothelium-dependent vasodilator responsiveness that is accomplished at a lower rate of NO synthesis in patients with coronary artery disease [9]. Clinical trials with ACEIs in patients with a low or preserved ejection fraction have documented a significant reduction in cardiovascular death, MI, stroke, and the need for coronary revascularization producers $[10,11]$. A recent meta-analysis with a pooled analysis of 20 cardiovascular morbidity-mortality trials demonstrated that ACEIs treatment was associated with a significant $10 \%$ reduction in all-cause mortality (HR, 0.90; 95\% CI, 0.84 to $0.97 ; p=0.004$ ), whereas no mortality reduction could be demonstrated with ARBs treatment (HR, 0.99; $95 \%$ CI, 0.94 to $1.04 ; p=0.683$ ). This difference in treat- ment outcome between ACEIs and ARBs on all-cause mortality was statistically significant (with a $p$ value for heterogeneity equal to 0.036 ) [12].

On the other hand, angiotensin II activates NF- $\mathrm{KB}$ induced by oxidative stress, mediated by the angiotensin II type $1\left(\mathrm{AT}_{1}\right)$ receptor [8]. We reported the pleiotropic effects of candesartan, one of the ARBs, in addition to its anti-hypertensive effect, that significantly improved flow-mediated vasodilation and reduced the biomarkers of oxidant stress, inflammation, and hemostasis in patients with hypertension, independently of blood pressure reduction [13]. Now, patients at high risk of cardiovascular events such as MI are required to take statins as a preventative measure [14]. It is rather surprising that only $4.5 \%$ and $2.9 \%$ of patients with ARBs and ACEIs, respectively, took lipid-lowering drugs prior to an acute MI [5]. Of particular note, experimental and clinical studies demonstrated a cross-talk between hypercholesterolemia and RAS at multiple stages. Therefore, statins and ACEIs or ARBs may have the potential to exert additive/ synergistic beneficial effects on both endothelial function and insulin sensitivity when compared with monotherapy in patients with cardiovascular risk factors by both distinct and interrelated mechanisms (Fig. 2) [15-19]. Recently, we observed that pravastatin combined with

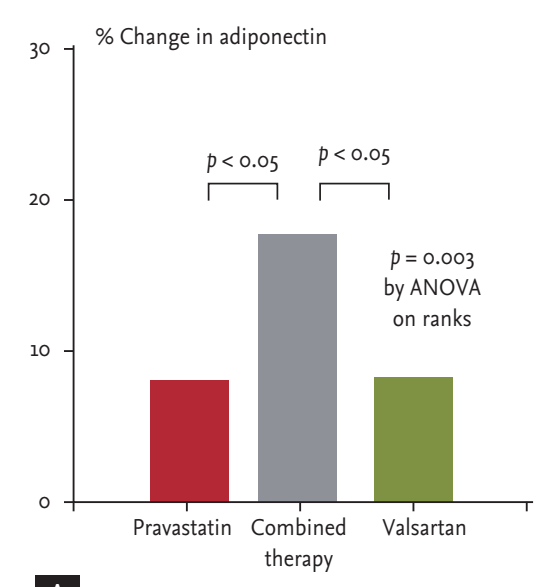

A

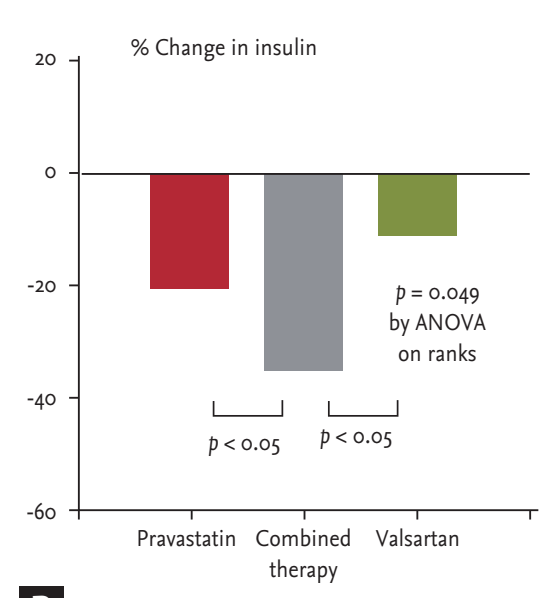

B

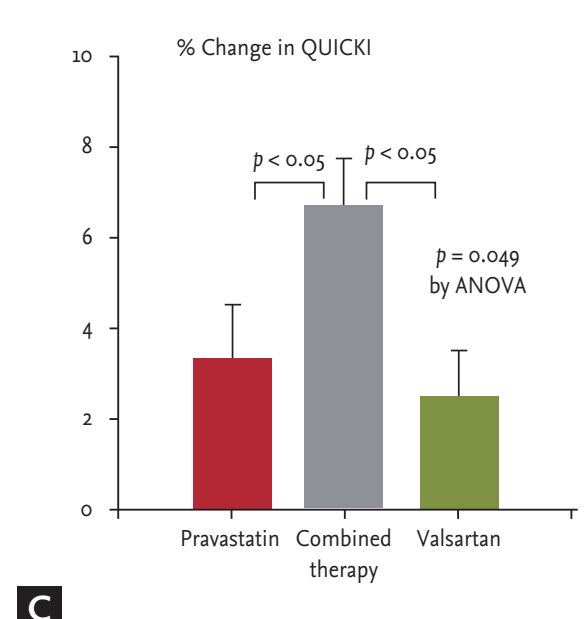

c

Figure 3. The synergistic effect of combination treatment with valsartan and pravastatin on insulin sensitivity. In 48 hypercholesterolemic patients, both pravastatin $40 \mathrm{mg}$ and valsartan $160 \mathrm{mg}$ increased plasma adiponectin levels, reduced fasting insulin levels, and increased insulin sensitivity relative to the baseline measurements. When pravastatin was combined with valsartan, their response increased in an additive manner when compared with monotherapy alone. Median values (A, B) or mean with SEM (C) are provided. Reproduced with permission from Koh et al. [18]. QUICKI, quantitative insulin sensitivity check index; ANOVA, analysis of variance. 
valsartan therapy improved flow-mediated vasodilation, reduced the level of C-reactive protein, a biomarker of inflammation, increased plasma adiponectin, lowered fasting insulin levels, and improved insulin sensitivity in an additive manner compared to monotherapy alone in an hypertensive population (Fig. 3) [18].

Although Lee et al. [5] reported that the beneficial effects of ARBs were comparable to, or even better than, ACEIs in Korean patients with acute MI, few studies have been reported in the literature, compared with ACEIs in such patients, especially without left ventricular dysfunction or heart failure. Furthermore, ACEIs are cheaper than ARBs, albeit not rare dry coughing. Therefore, current guidelines recommend the initial use of ACEIs in such patients, changing to ARBs when patients become intolerant of ACEIs $[1,2]$.

\section{Conflict of interest}

No potential conflict of interest relevant to this article was reported.

\section{Acknowledgments}

K.K.K. holds a certificate of patent, 10-1579656.

\section{REFERENCES}

1. Task Force on the management of ST-segment elevation acute myocardial infarction of the European Society of Cardiology (ESC), Steg PG, James SK, et al. ESC Guidelines for the management of acute myocardial infarction in patients presenting with ST-segment elevation. Eur Heart J 2012;33:2569-2619.

2. O'Gara PT, Kushner FG, Ascheim DD, et al. 2013 ACCF/ AHA guideline for the management of ST-elevation myocardial infarction: a report of the American College of Cardiology Foundation/American Heart Association Task Force on Practice Guidelines. Circulation 2013;127:e362-e425.

3. Strauss MH, Hall AS. Angiotensin receptor blockers may increase risk of myocardial infarction: unraveling the ARB-MI paradox. Circulation 2006;114:838-854.

4. Tsuyuki RT, McDonald MA. Angiotensin receptor blockers do not increase risk of myocardial infarction. Circulation 2006;114:855-860.

5. Lee JH, Bae MH, Yang DH, et al. Angiotensin II type 1 receptor blockers as a first choice in patients with acute myocardial infarction. Korean J Intern Med 2016;31:267276.

6. Yang JH, Hahn JY, Song YB, et al. Angiotensin receptor blocker in patients with ST segment elevation myocardial infarction with preserved left ventricular systolic function: prospective cohort study. BMJ 2014;349:g6650.

7. Koh KK, Quon MJ, Han SH, Chung WJ, Kim JA, Shin EK. Vascular and metabolic effects of candesartan: insights from therapeutic interventions. J Hypertens Suppl 2006;24:S31-S38.

8. Koh KK, Oh PC, Quon MJ. Does reversal of oxidative stress and inflammation provide vascular protection? Cardiovasc Res 2009;81:649-659.

9. Koh KK, Bui MN, Hathaway L, et al. Mechanism by which quinapril improves vascular function in coronary artery disease. Am J Cardiol 1999;83:327-331.

10. Yusuf S, Pepine CJ, Garces C, et al. Effect of enalapril on myocardial infarction and unstable angina in patients with low ejection fractions. Lancet 1992;340:1173-1178.

11. Bosch J, Lonn E, Pogue J, et al. Long-term effects of ramipril on cardiovascular events and on diabetes: results of the HOPE study extension. Circulation 2005;112:13391346.

12. van Vark LC, Bertrand M, Akkerhuis KM, et al. Angiotensin-converting enzyme inhibitors reduce mortality in hypertension: a meta-analysis of randomized clinical trials of renin-angiotensin-aldosterone system inhibitors involving 158,998 patients. Eur Heart J 2012;33:20882097.

13. Koh KK, Ahn JY, Han SH, et al. Pleiotropic effects of angiotensin II receptor blocker in hypertensive patients. J Am Coll Cardiol 2003;42:905-910.

14. Kim JW, Yun KH, Kim EK, et al. Effect of high dose rosuvastatin loading before primary percutaneous coronary intervention on infarct size in patients with ST-segment elevation myocardial infarction. Korean Circ J 2014;44:76-81.

15. Lim S, Sakuma I, Quon MJ, Koh KK. Potentially important considerations in choosing specific statin treatments to reduce overall morbidity and mortality. Int J Cardiol 2013;167:1696-1702.

16. Koh KK, Sakuma I, Hayashi T, Kim SH, Chung WJ. Renin-angiotensin system inhibitor and statins combination therapeutics: what have we learnt? Expert Opin Pharmacother 2015;16:949-953. 
17. Koh KK, Quon MJ, Han SH, et al. Vascular and metabolic effects of combined therapy with ramipril and simvastatin in patients with type 2 diabetes. Hypertension 2005;45:1088-1093.

18. Koh KK, Lim S, Choi H, et al. Combination pravastatin and valsartan treatment has additive beneficial effects to simultaneously improve both metabolic and cardio- vascular phenotypes beyond that of monotherapy with either drug in patients with primary hypercholesterolemia. Diabetes 2013;62:3547-3552.

19. Jang JY, Lee SH, Kim BS, et al. Additive beneficial effects of valsartan combined with rosuvastatin in the treatment of hypercholesterolemic hypertensive patients. Korean Circ J 2015;45:225-233. 